\title{
Conserved motifs in Fos and Jun define a new class of activation domain
}

\author{
Jacqueline A. Sutherland, Alistair Cook, Andrew J. Bannister, and Tony Kouzarides ${ }^{1}$ \\ Wellcome/CRC Institute, University of Cambridge, Cambridge, CB2 IQR UK
}

Fos and Jun form a tight heterodimeric complex that activates transcription by AP1 sites. We have recognized that two adjacent regions of the Jun A1 activation domain are conserved in the Fos protein, and we refer to these two homologous regions as homology box 1 (HOB1) and homology box 2 (HOB2). Using GAL4 chimeras, we show that the HOB1/HOB2 region of Fos and Jun is an independent activation domain in which HOB1 and $H O B 2$ act cooperatively to activate transcription. This cooperativity is retained after the replacement of Fos HOB1 or HOB2 with the equivalent domain of Jun or when duplicated HOB1/HOB1 and HOB2/HOB2 combinations are generated. In the Fos protein, HOB1 or HOB2 can also cooperate with a distinct domain at the carboxyl terminus of the protein. Using the НОВ2 consensus sequence as a guide, we identified a HOB2-containing activation domain in the CCAAT/enhancer binding protein (C/EBP) protein. This HOB2 motif can cooperate with as yet undefined sequences in C/EBP and will function even when linked to Jun HOB1. Thus, HOB1 and HOB2 represent inert "cooperating modules" that are combined to generate a functional activation domain. Each of these modules has the potential to cooperate with both distinct and identical domains. The presence of HOB-like modules in three different transcription factors indicates that the HOB motifs characterize a new class of activation domain. These motifs can be used now to identify other transcription factors with such modular characteristics.

[Key Words: Transcription; activation; Fos; Jun; C/EBP]

Received March 3, 1992; revised version accepted June 11, 1992.

The transcription factors Fos and Jun can regulate the expression of genes bearing AP1 sites. These two proteins share a common basic leucine zipper (bZIP) DNAbinding domain (Landschulz et al. 1988a) that allows them to dimerize by the leucine zipper and bind DNA by a basic motif (Halazonetis et al. 1988; Kouzarides and Ziff 1988; Nakabeppu et al. 1988; Sassone-Corsi et al. 1988; Gentz et al. 1989; Schuermann et al. 1989; Turner and Tjian 1989). Each of these two proteins belongs to a different family of either Fos- or Jun-related proteins. Members of each family share a number of conserved domains, but the only recognized homology between the Fos family (Fos, Fra-1, Fra-2, Fos-B) and the Jun family (Jun, Jun-B, Jun-D) is within their DNA-binding domain. The leucine zipper structure within this domain provides the specificity of interaction between Fos and Jun family members (Kouzarides and Ziff 1989a; Sellers and Struhl 1989). All Fos-related proteins can form functional, DNA-binding heterodimers with any of the Junrelated proteins, suggesting that a variety of Fos- and Jun-related heterodimers, with potentially different effector domains, may be present in the cell (for review, see Kouzarides and Ziff 1989b). The Jun family members can also form homodimers and heterodimers with each other, whereas the Fos family members can only het-

${ }^{1}$ Corresponding author. erodimerize with members of the Jun family. The role of the Fos- and Jun-related proteins and how this role differs from that of Fos and Jun is unclear, but some clues do exist. For example, unlike Jun, Jun-B is incapable of activating the transcription of a promoter bearing a single AP1 site and can inhibit the activation of such a promoter by the Jun protein (Chiu et al. 1989; Schütte et al. 19891.

Once bound to DNA, the Fos-Jun complex can regulate gene expression by virtue of specific activation domains carried by each protein (Angel et al. 1989; Abate et al. 1990). Structure-function analysis of the Jun protein has identified several transcriptional activation domains in assays both in vitro (Bohmann and Tiian 1989) and in vivo (Angel et al. 1989; Baichwal and Tjian 1990). A major determinant appears to be at the amino terminus of the protein (Angel et al. 1989; Baichwal and Tjian 1990), whereas a relatively weak activation domain is present toward its carboxyl terminus (Baichwal and Tjian 1990). Various lines of evidence suggest that the ability of the amino-terminal domain to activate is regulated both positively and negatively. Evidence for negative regulation comes from in vivo competition experiments that show that a cell type-specific inhibitor interacts with this domain and represses its activity (Baichwal and Tjian 1990). The target for this down-regulation appears to be (at least partly) a 27-residue domain, called the $\delta$ region, 
which is found to be deleted in the v-jun oncogene. Positive regulation of this activation domain is mediated by certain activated oncogene products. Cotransfection of $\mathrm{H}$-ras or v-src expression vectors stimulates the activation potential of this Jun domain (Baichwal et al. 1991; Binétruy et al. 1991). The stimulatory effect of H-ras may be a direct consequence of Jun phosphorylation, as two serine residues within the Jun activation domain are specifically phosphorylated in response to H-ras (Binétruy et al. 1991; Smeal et al. 1991).

Here we show that Fos and Jun have previously unrecognized homology outside their bZIP DNA-binding domain. This homology represents the conservation of two adjacent regions, homology box 1 (HOB1) and homology box 2 (HOB2), that fall within their activation domain. Using GAL4 DNA-binding domain chimeras, we show that HOB1 and HOB2 function cooperatively to activate transcription. In addition, they each cooperate with a distinct domain in the Fos carboxyl terminus and can function even when repeated in a $\mathrm{HOB} 1 / \mathrm{HOB} 1$ or HOB2/HOB2 combination. Our results establish the existence of inert "cooperating modules" that activate transcription when combined with a second /identical or distinct) module.

\section{Results}

Fos shows homology to a Jun activation domain

Given that Fos and Jun cooperate to activate transcription, we reasoned that Fos and Jun may possess common trans-activating domains, in addition to a common DNA-binding domain. Because comparison of the primary amino acid sequence did not reveal any obvious homology outside their DNA-binding domain, we compared the secondary structure predictions of the two proteins. This type of analysis focused our attention on two adjacent, potentially helical regions of Jun shown to be required for the transcriptional integrity of the Jun homodimer (Angel et al. 1989; Baichwal et al. 1990). These two regions of Jun show homology to two identically spaced, potentially helical regions of the Fos protein (Figs. 1 and 2). We refer to these two homologous regions as $\mathrm{HOB} 1$ and $\mathrm{HOB} 2$. In the Jun protein, HOB1 and HOB2 are present near the amino terminus, within the $\mathrm{Al}$ activation domain (Baichwal and Tjian 1990). Deletion analysis of Jun has shown that domains covering precisely the $\mathrm{HOB} 1$ and $\mathrm{HOB} 2$ sequences are essential for Jun-induced transcriptional activation (Angel et al. 1989; Fig. 1). In Fos, $\mathrm{HOB} 1$ and $\mathrm{HOB} 2$ are present after the leucine zipper region, near the carboxyl terminus of the protein (Fig. 1).

\section{Transcriptional activation domains of Fos}

We set out to establish whether the HOB1/HOB2 region of Fos possesses transcriptional activation functions, as in the case of the HOB1/HOB2 region of Jun. Because Fos is unable to bind DNA on its own, we fused the Fos protein to the DNA-binding domain of the yeast transcription factor GAL4 and used this chimera (GF 1-380) to activate transcription of a promoter-bearing GAL4binding site (Fig. 3). Deletion analysis of GF 1-380 indicates that a region containing both HOB1 and HOB2 (amino acids 210-308) is a major contributor to Fos transcriptional activity (cf. lanes 2 and 5) and that sequences at the amino terminus of the protein also possess transcriptional activation functions (cf. lanes 1 and 3). The HOB1/HOB2 region (amino acids 210-308) appears to have independent trans-activating ability in the absence of the rest of the Fos protein, as it can activate transcription very efficiently when fused to the GAL4 DNA-binding domain (lane 6). When a fragment containing only

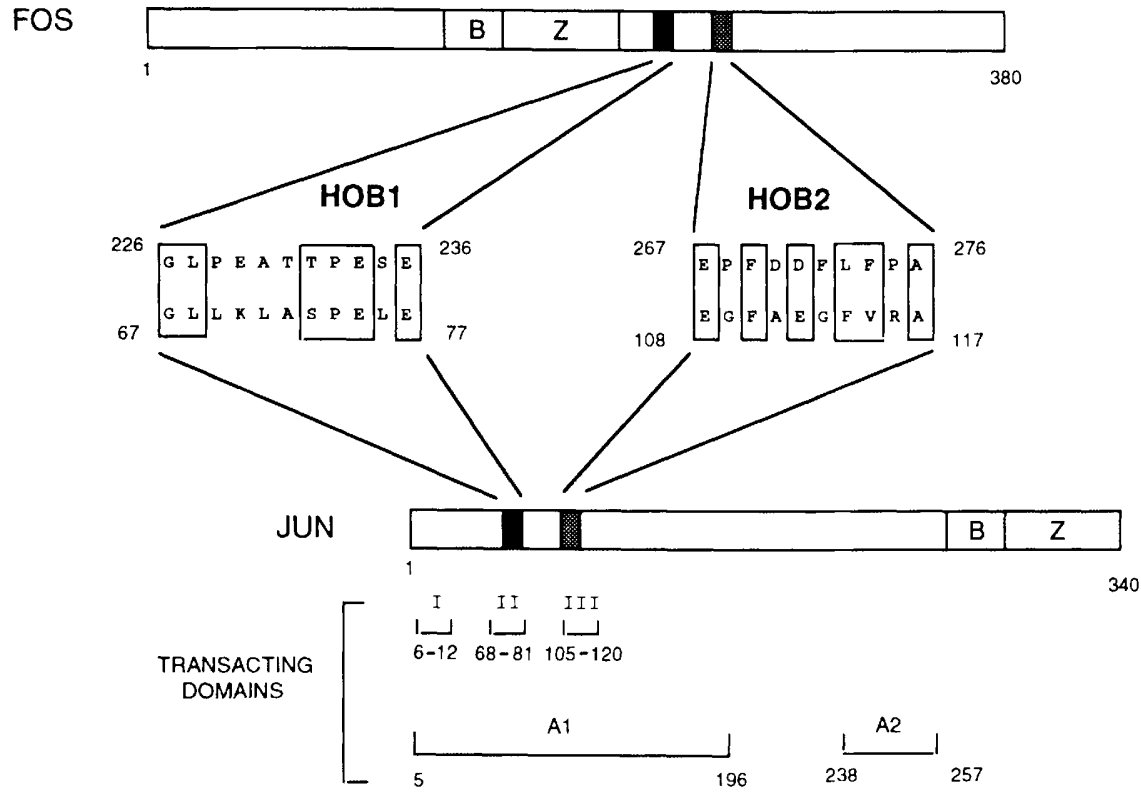

Figure 1. The HOBI and HOB2 motifs of Fos lie within a trans-activating domain. Diagrammatic representation of the Fos and Jun proteins shows the position and alignment of $\mathrm{HOB} 1$ and $\mathrm{HOB} 2$. The previously described in vivo activation regions of Jun are shown below the Jun protein. Regions I, II, and III were described by Angel et al. (1989) and regions A1 and A2 were reported by Baichwal and Tjian (1990). The HOB1 and HOB2 motifs of Jun lie within trans-activating regions II and III, respectively. The position of the Fos and Jun basic DNA-binding motif $(B)$ and leucine zipper dimerization motif $(Z)$ is indicated. 
A

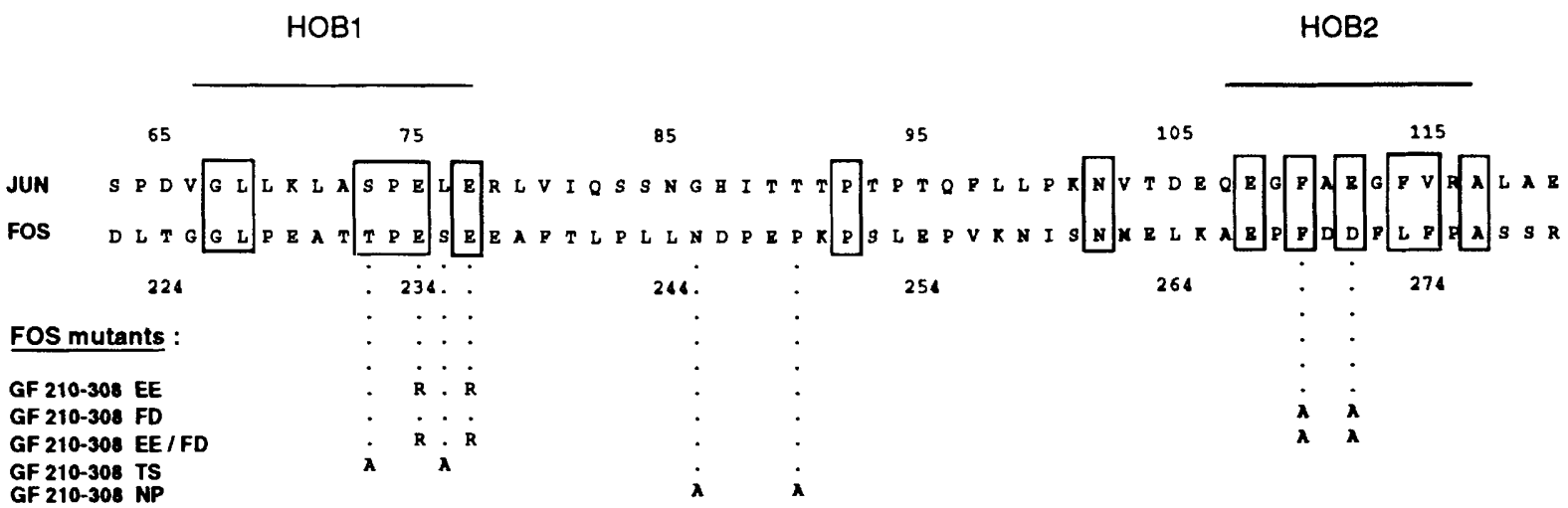

B

Figure 2. Residues within $\mathrm{HOB} 1$ and $\mathrm{HOB} 2$ contribute to Fos-induced transcriptional activation. (A) Alignment of the rat c-Fos (Curran et al. 1987) and the human c-Jun (Angel et al. 1988) regions that span $\mathrm{HOB} 1$ and $\mathrm{HOB} 2$, showing the precise conservation of distance between the HOB1 and HOB2 in Fos and Jun. Below the alignment, the mutations introduced within the Fos sequence are shown. $(B)$ The GF 210-308 plasmid (wt) and the various mutants of this (shown in $A$ ) were introduced into $1 \mathrm{BR}$ cells along with the pUAS10CAT reporter plasmid as described in Fig. 3. CAT activity of the GF 210-308 plasmid (wt) was arbitrarily set at 100 , and the values for the various mutants are shown relative to this.

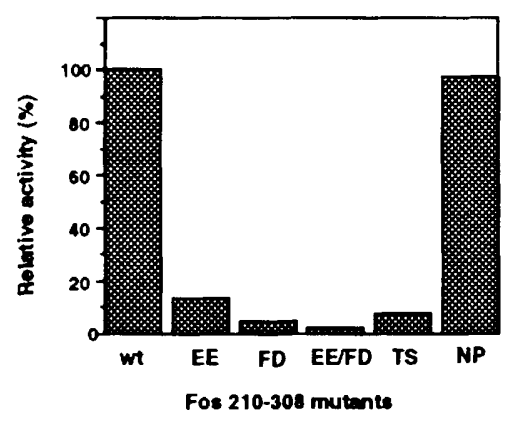

HOB1 (GF 210-244, lane 7) or only HOB2 (GF 250-308, lane 8 | is used, however, no activation is detected. This result is consistent with the notion that $\mathrm{HOBl}$ and HOB2 are interdependent for transcriptional activity.

Our deletion analysis also suggests the presence of an additional trans-activating domain carboxy-terminal to HOB2. A chimera that contains the HOB1/HOB2 region plus the remaining Fos carboxyl terminus (GF 210-380, lane 9) shows higher activity than GF 210-308, which contains the HOB1/ HOB2 region alone. This carboxyterminal domain is unable to function in the context of a larger protein (cf. lanes 2 and 4 ), however, probably owing to the presence of a repressor function elsewhere in the protein. A repressor function must also affect the activity of the HOB1/HOB2 region as this domain shows higher activity when amino-terminal sequences are removed (cf. lanes 4 and 6). The presence of repressive domains within the Fos protein is consistent with recent in vitro results from Abate et al. (1991). These studies also show that the region covering the Fos HOB1/HOB2 domain can function in vitro in the context of the fulllength Fos-Jun complex.

\section{Residues within Fos $H O B 1$ and $H O B 2$ contribute to} trans-activation

Having shown that a 99-amino-acid region (210-308) encompassing the Fos $\mathrm{HOB} 1$ and HOB2 motifs can func- tion as an independent trans-activating domain, we sought to verify that residues within HOB1 and HOB2 contribute to the activity exhibited by this region. First, we chose to mutate two glutamic acid residues [E-234 and E-236) in Fos HOB1 (Fig. 2A). Substitution of the corresponding residues ( $\mathrm{E}-75$ and $\mathrm{E}-77$ ) in Jun to arginine (R) results in a reduction of Jun trans-activating ability (Angel et al. 1989). Figure 2B shows that mutating Fos E-234 and E-236 (EE) to arginine reduces trans-activation by over sevenfold. In contrast, mutating two residues within the nonconserved region between HOB1 and HOB2 (NP) has no effect on trans-activation. This supports the argument that homologous residues within the Fos and Jun HOB1 motif are functionally related. Inspection of the HOB1 motif suggested to us that these two $E$ residues may form part of a recognition site for a protein kinase, as an S/T residue (73 in Jun and 232 in Fos) is conserved in the vicinity. We therefore mutated Fos $\mathrm{T}-232$ and an additional serine residue, $\mathrm{S}-235$, to alanine and found that this double mutation, TS, also affects trans-activation. This result is consistent with the possibility that a phosphorylation event may regulate the activity of this region.

To assess the contribution of the HOB2 motif, we mutated two conserved residues, F-269 and D-271, to alanine. As seen in Figure 2B, the FD mutant is severely defective in trans-activating ability compared with the wild-type GF 210-308 (20-fold lower activity). This 
A

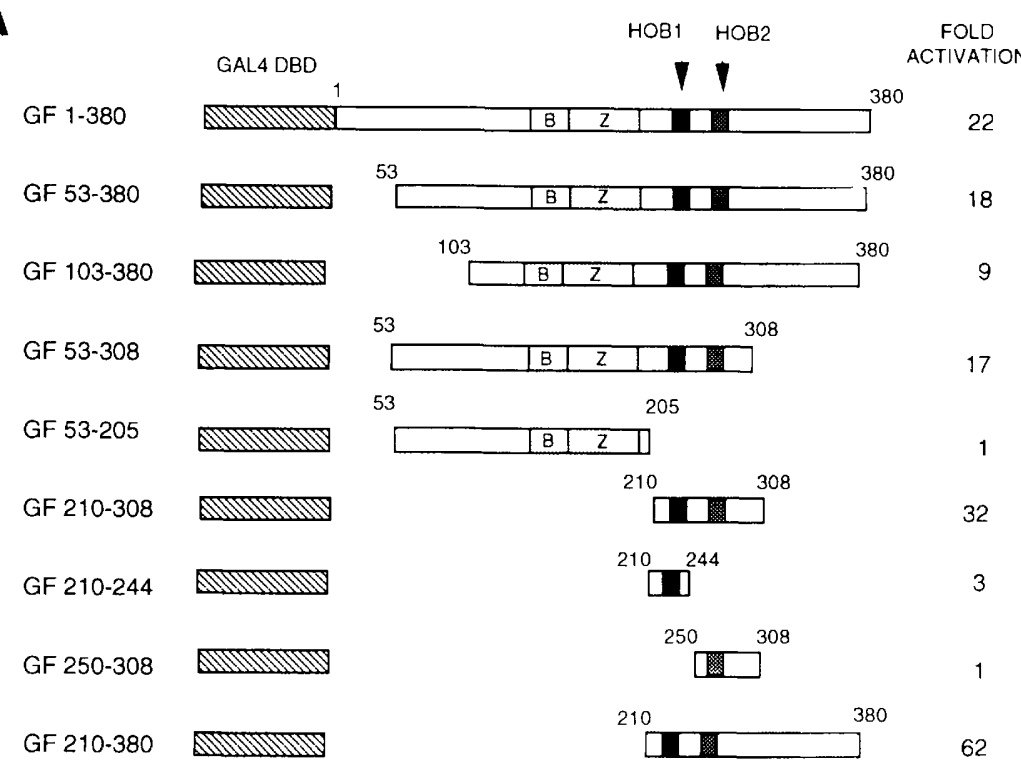

B

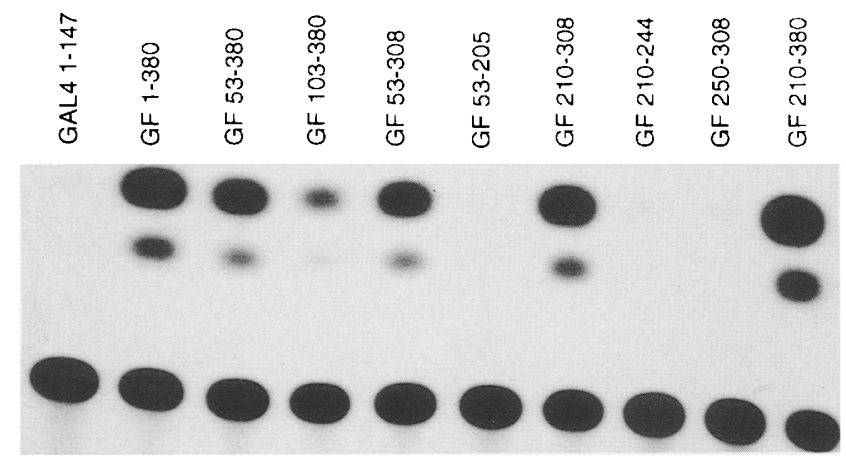

Figure 3. Identification of transcriptional activation domains within the Fos protein. (A) Schematic representation of the Fos deletion analysis. Deletions of the Fos protein are fused to the GAL4 DNA-binding domain (DBD). All constructs are in an SV40 early promoter expression vector. The activity of each fusion is shown as an increase in CAT activity above the level induced by the GAL4 DBD alone. Values represent an average of several independent transfections. The $\mathrm{HOB} 1$ and $\mathrm{HOB} 2$ motifs (black and stippled boxes) as well as the basic (B) and zipper $(Z)$ regions are shown. The GAL4 DBD is shown as a box with diagonal lines. $(B)$ Activity of the GAL4-Fos deletions. Four micrograms of the target reporter plasmid pUAS10CAT (containing GAL4-binding sites; Cousens et al. 1989) was cotransfected with 1 $\mu \mathrm{g}$ of the SV40 promoter-expressed GAL4-Fos chimeras (shown in A) into l BR cells. Extracts from these cells were used for CAT assays and Western blots. The Western blots were probed with an antibody against the GAL4 DBD to verify that each plasmid expressed equivalent levels of protein.
HOB2 mutant shows less activity than the HOB1 mutant $\mathrm{EE}$, whereas the double mutant EE/FD shows marginally less activity than either. These results verify that both $\mathrm{HOB} 1$ and HOB2 contribute to the transcriptional activity exhibited by this region.

Fos and Jun have functionally interchangeable $H O B 1$ and HOB2 domains

Having established that Fos HOB1 and HOB2 only function in combination (Fig. 3), we asked whether either of these motifs could be replaced by the corresponding HOB motif of Jun. Figure 4 shows that a domain of Jun that contains both HOB1 and HOB2 (lane 4) can activate transcription when linked to the GAL4 DNA-binding domain, whereas a region containing HOB1 (lane 5) or HOB2 (lane 6) alone is essentially inactive. This parallels the results obtained with Fos, where HOB1 and HOB2 together activate transcription (lane 1), whereas HOB1 or HOB2 alone do not (lanes 2,3). If we now make a chimera containing Fos HOB1 linked to Jun HOB2 $(\mathrm{GFH} 1 / \mathrm{JH} 2$, lane 7) or Jun HOB1 linked to Fos HOB2 (G)H1/FH2, lane 8) we can restore activity. The cooperativity between these Fos and Jun domains is dependent on an intact HOB motif, as mutagenesis of Fos HOB2 $\{E E\rangle$ or Fos HOBI (FD) severely reduces this cooperativity. These data suggest that the HOB1/HOB2 motifs of Fos and Jun are operationally equivalent and provide strong evidence that the HOB1 and HOB2 domains activate transcription cooperatively.

Fos $H O B 1$ or HOB2 can cooperate with carboxy-terminal sequences

Our analysis of the HOB1/HOB2 domain of Fos (see Fig. 3) revealed that an additional activation domain may be present within the carboxyl terminus of the Fos protein (Fig. 5, cf. lanes 1 and 2). Having established that HOB1 and $\mathrm{HOB} 2$ are cooperating units, we asked whether this additional carboxy-terminal activity was reliant on the presence of HOB1 or HOB2 or both. Figure 5 shows that the carboxyl terminus of Fos (sequences precisely carboxy-terminal to HOB2, GF 276-380, lane 6) has no independent trans-activating ability. When the inactive 
Figure 4. The HOB1 or HOB2 domains of Jun can be functionally replaced by the equivalent $\mathrm{HOB}$ domain of Fos. (A) Schematic representation of the GAL4 DBD fusions containing various combinations of Fos HOB1 (FH1), Fos HOB2 (FH2), Jun HOB1 (JH1), and Jun HOB2 (JH2). The EE and FD mutations are as shown in Fig. 2. The values on the right represent the increase in CAT activity induced by each construct, above the level exhibited by the GAL4 DBD alone. (B) Activity of "domainswapped" chimeras. The chimeric constructs shown in $A$ expressed under an SV40 promoter were cotransfected with the pUAS10CAT reporter into $1 \mathrm{BR}$ cells as described in Fig. 3.
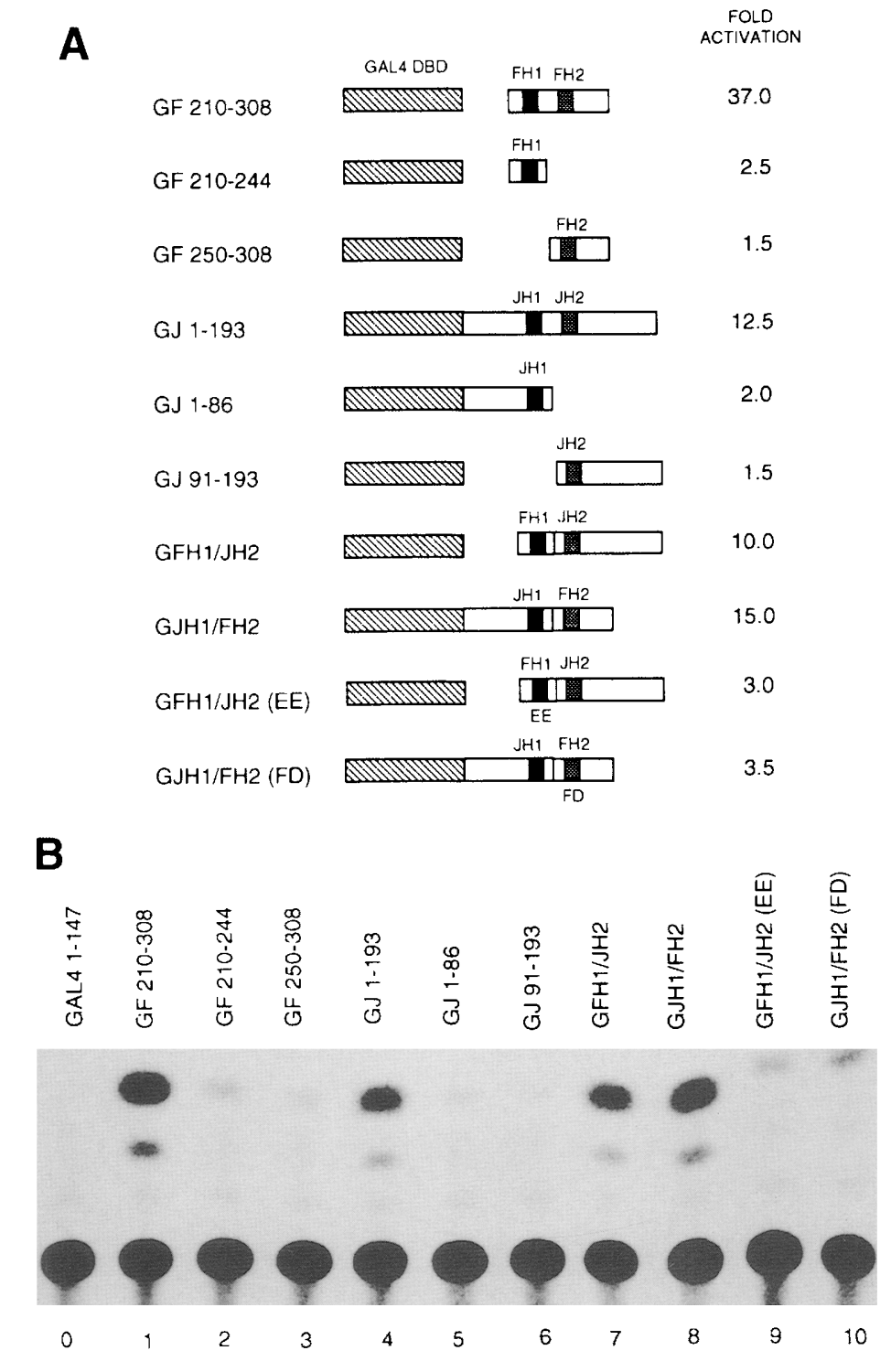

HOB1 or HOB2 domain is linked to the carboxyl terminus, however, very efficient trans-activation is observed (GF $\Delta 210-380$, lane 4 and GF 250-380, lane 3). This indicates that HOB1 and HOB2 can cooperate equally well with a distinct domain present at the carboxyl terminus. The presence of both HOB1 and HOB2 along with the carboxyl terminus does not increase cooperativity but shows additive behavior (GF 210-380, lane 1). Thus, the higher activity of GF 210-380 compared with GF 210-308 is due to cooperation between both $\mathrm{HOBl}$ and HOB2 with the carboxy-terminal domain.

The domain cooperating with HOB2 appears to lie within the carboxy-terminal 48 residues of Fos. Deletion of these sequences (GF 250-332) severely reduces the trans-activating potential of GF 250-380. Because these 48 carboxy-terminal residues do not show significant trans-activating potential (GF 314-380, lane 8), we conclude that the very carboxyl terminus of Fos must con- tain an inert domain that has the capacity to activate transcription in cooperation with HOB2.

\section{A functional $H O B 2$ domain in $C / E B P$}

The presence of the HOB1 and HOB2 motifs in members of two different transcription factor families prompted us to look for the presence of these motifs in the transactivating domains of other transcription factors. The search for HOB1 was not fruitful, but the search for HOB2 homologies proved more conclusive (Fig. 6). This motif was found within the activation domain of the CCAAT/enhancer binding protein (C/EBP) transcription factor. Friedman and McKnight (1990) have shown that a 29-residue region of C/EBP (which contains the HOB2 motif) is unable to activate transcription when linked to the GAL4 DNA-binding domain but can do so very efficiently when amino-terminal sequences with no intrin- 
A

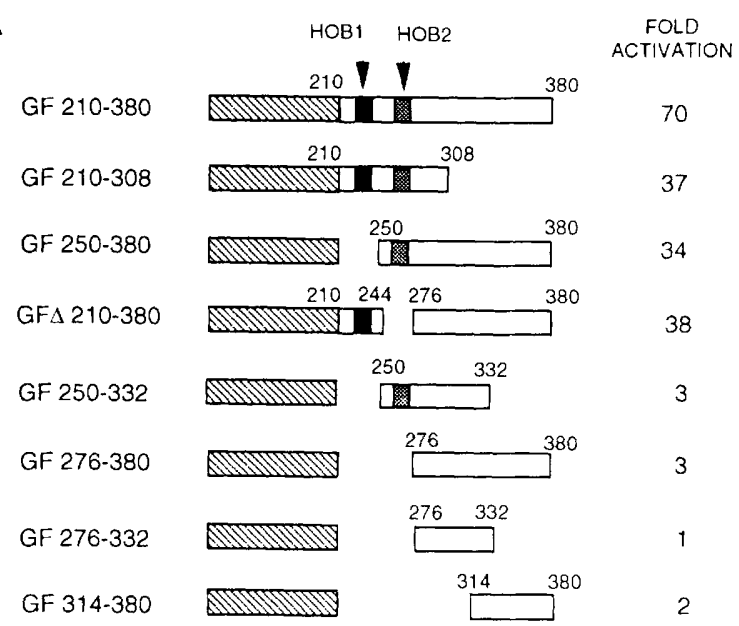

B

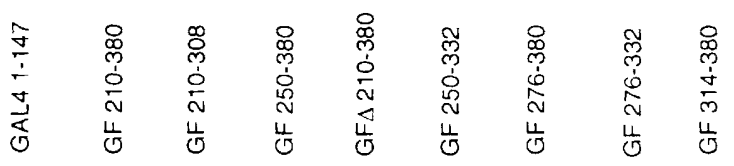

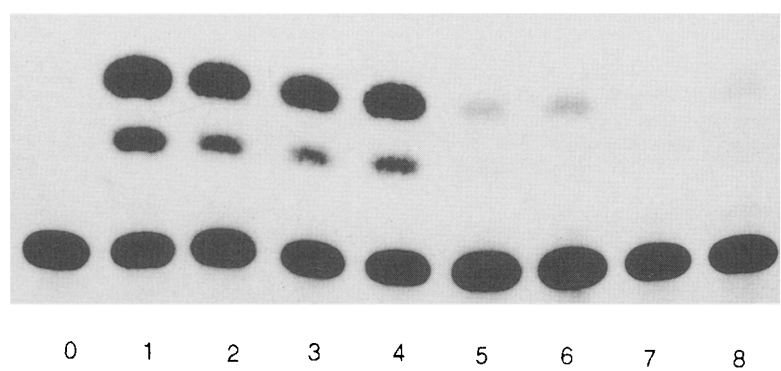

Figure 5. Fos HOB2 cooperates with the carboxyl terminus. $(A)$ Schematic representation of GAL4 DBD fusions containing portions of the Fos carboxyl terminus $\{210-380\}$. The values on the right represent the increase in CAT activity induced by each construct, above the level exhibited by the GAL4 DBD alone. $|B|$ CAT activity of each construct shown in $A$. The chimeras were expressed under an SV40 promoter and cotransfected with the pUAS10CAT reporter into 1 BR cells as described in Fig. 3.

sic activation functions are linked to it. This precisely parallels the characteristics of the Fos and Jun HOB2 motif, where an adjacent amino-terminal domain (containing $\mathrm{HOB} 1\}$ is required for its activity.

We therefore asked whether the C/EBP HOB2 motif can functionally replace the HOB2 motif of Jun. Figure 7 shows that this 29 -residue C/EBP domain, containing the HOB2 motif, cooperates with the HOB1 domain of Iun (cf. lanes 3 and 4). In agreement with previous observations (Friedman and McKnight 1990), this 29-residue region cannot activate transcription independently (lane 2) but can do so when amino-terminal C/EBP residues are present (lane 1). We can find no obvious HOB1 motif in this region of C/EBP or any homology to the carboxyl terminus of Fos. Sequences unrelated to these two domains or ones with limited homology must be cooperating with C/EBP HOB2.
Duplication of HOB1 or HOB2 generates an activation domain

The synergistic effect on transcription exhibited by HOB1 and HOB2 suggests that this activation domain has a modular structure. Next, we asked whether this modularity is a unique feature of the $\mathrm{HOB} 1 / \mathrm{HOB} 2 \mathrm{com}-$ bination or whether modularity is an intrinsic characteristic of each of these two motifs. If the latter is correct we would expect synergistic behavior from a duplication of either HOB1 or HOB2. Figure 8 shows that a duplicated version of Fos HOB1 (GFH1/H1) or HOB2 (GFH2/ $\mathrm{H} 2$ ) can activate transcription very efficiently compared with an essentially inactive single copy of HOB1 or HOB2. The activity of each dimerized domain is 14-fold above that of the single copy (sevenfold above that expected for additive behavior), indicating synergism between identical HOB domains. In contrast, duplication of the highly active $\mathrm{HOB} 1 / \mathrm{HOB} 2$ domain $\left[\mathrm{GF}(\mathrm{Hl} / \mathrm{H} 2)_{2}\right]$ only gives an essentially additive, 2.5 -fold increase in activity (Fig. 8, cf. lanes 1 and 6).

These results suggest that $\mathrm{HOB} 1$ and $\mathrm{HOB} 2$ represent distinct activation modules within the $\mathrm{HOB} 1 / \mathrm{HOB} 2$ domain. These modules have no intrinsic trans-activating ability but have the potential to form a strongly transactivating domain when combined with a second /identical or distinct) activation module. Synergism between activation modules is limited to the generation of a single active domain, as further synergism is not observed when two copies of such an active domain are linked.

\section{Discussion}

Sequence homology between a Jun activation domain and an undefined region of the Fos protein has led to the identification of a novel class of activation domain. In vivo assays with GAL4 DBD chimeras have allowed us to compare directly the activation potential of Fos and Jun sequences containing the homologous regions HOB1 and HOB2. The results clearly show that (1) domains containing $\mathrm{HOB} 1$ or $\mathrm{HOB} 2$ have almost no activation capacity but can work cooperatively to activate transcription; (2) the HOB motifs in Fos and Jun are functionally interchangeable; therefore, chimeras consisting of Fos HOB1/Jun HOB2 or Jun HOB1/Fos HOB2 form functional activating domains; (3) HOB1 and HOB2 can activate transcription synergistically even when present in a repeating $\mathrm{HOB} 1 / \mathrm{HOB} 1$ or $\mathrm{HOB} 2 / \mathrm{HOB} 2$ combination, (4) Fos HOB1 or HOB2 can cooperate with a distinct sequence at the Fos carboxyl terminus; and (5) a functional HOB2 motif, capable of cooperating with Jun HOBl, is present in the C/EBP protein.

These properties, exhibited by HOB 1 and HOB2, demonstrate the existence of inert "activation modules." Such modules can activate transcription cooperatively with a second activation module, which can be either identical to itself or of a distinct class. The use of activation modules may reflect a widely used principle for the generation of activation domains. We have identified three different transcription factors that possess such 
Figure 6. The HOB2 motif shows homology to a trans-activating domain of C/EBP. The homologous residues are boxed. The residues predicted to be within an $\alpha$-helical conformation are marked by an $\mathrm{x}$ under the amino acid sequence. The phosphorylation of Ser residues (S-63 and S-73) in Jun (marked by an *) is stimulated by H-ras and is carried out in vitro by MAP kinase (Binétruy et al. 1991; Pulverer et al. 1991). Mutagenesis of these two serine residues drastically reduces Jun-mediated trans-activation (Pulverer et al. 1991). The sequence of C/EBP is from Landschulz et al. (1988b).

$$
\begin{aligned}
& \text { HOB1 } \\
& \text { НОВ2 }
\end{aligned}
$$

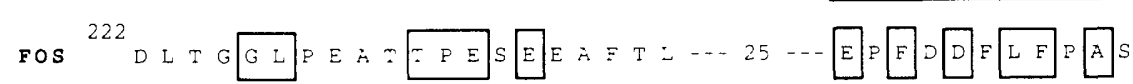

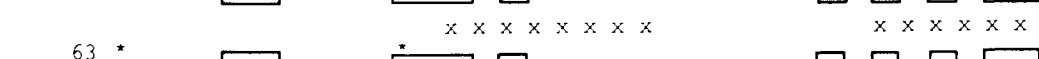

$$
\begin{aligned}
& \text { JUN S S P D V G L K L } A \text { S F E E ER L I I Q } \\
& \text { C/BBP }
\end{aligned}
$$

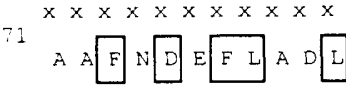

modules, and we have shown that modules belonging to different proteins can cooperate. It would be interesting to establish whether other already characterized activation domains are composed of such nonfunctional but cooperative units and whether these units can cooperate with HOB1 or HOB2. A domain with characteristics reminiscent of an activation module has been identified within the glutamine activation domain of OCT1. A small unit from this domain shows highly synergistic properties upon multimerization (W. Herr and $M$. Tanaka, pers. comm.).

A

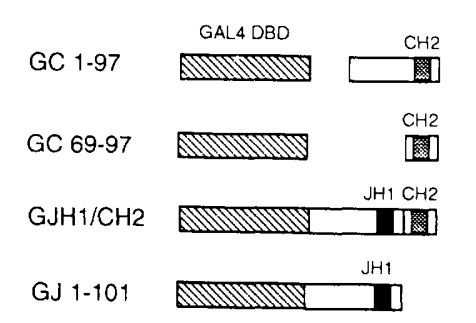

B

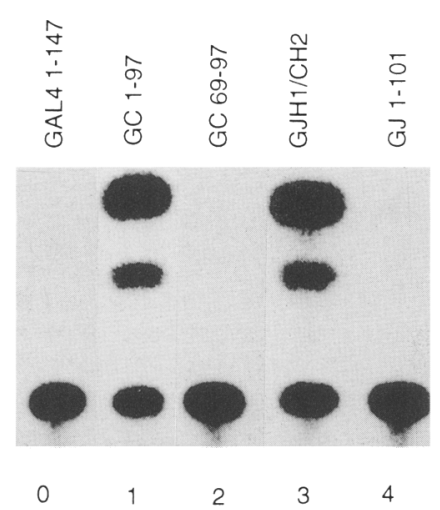

Figure 7. $\mathrm{C} / \mathrm{EBP}$ contains a cooperating $\mathrm{HOB} 2$ domain. $(A)$ Schematic representation of the GAL4 DBD fusions containing various combinations of $\mathrm{C} / \mathrm{EBP}$ HOB2 $(\mathrm{CH} 2)$ and Jun HOB1 (JHI) domains. The activity of each chimera is shown on the right as the increase in CAT activity above the level exhibited by the GAL4 DBD alone. (B) The CAT activity of each chimera shown in $A$. The constructs, expressed under an SV40 promoter, were cotransfected with the pUAS10CAT reporter into $1 \mathrm{BR}$ cells as described in Fig. 3.
A number of recent observations regarding the $\mathrm{Al}$ activation domain of Jun suggest that the transcriptional activity of the $\mathrm{HOB} 1 / \mathrm{HOB} 2$ domain is regulated by

A

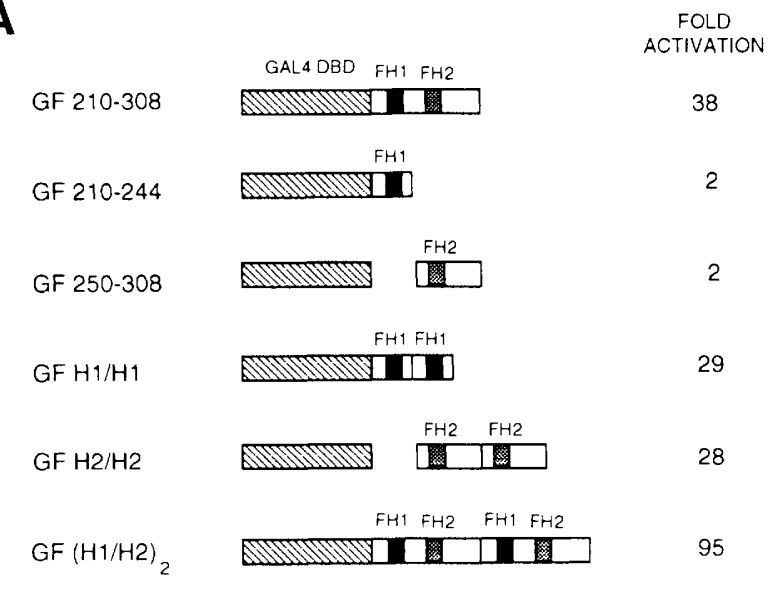

B

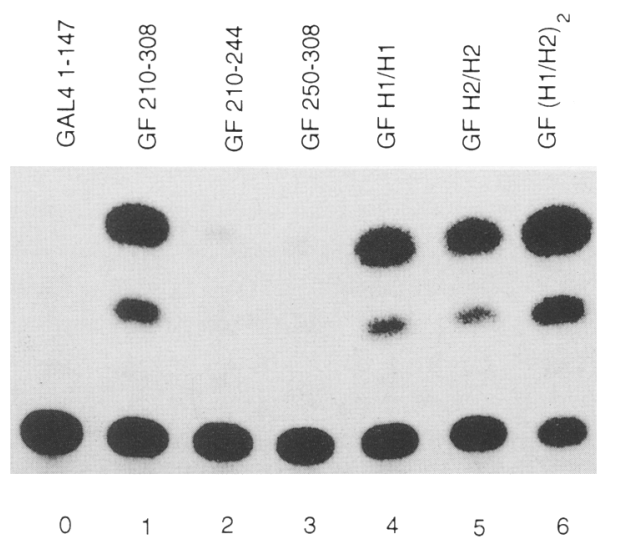

Figure 8. HOB1 or HOB2 function when duplicated. $(A)$ Schematic representation of GAL4 DBD fusions containing various combinations of Fos HOBl (FH1) and Fos HOB2 (FH2). The values on the right represent the increase in CAT activity induced by each construct, above the level exhibited by the GAL4 DBD alone. $(B)$ CAT activity of each construct shown in $A$. The chimeras were expressed under the SV 40 promoter and cotransfected with pUASIOCAT reporter into 1 BR cells, as described in Fig. 3. 
phosphorylation events within HOB1. The transcriptional activity of the Jun Al domain is augmented by the $\mathrm{H}$-ras protein. This "superactivation" process is reliant on the phosphorylation of two serine residues, S-63 and $\mathrm{S}-73$, which is induced by $\mathrm{H}$-ras in vivo and can be carried out by MAP kinase in vitro (Binétruy et al. 1991; Pulverer et al. 1991; Smeal et al. 1991). Interestingly, Jun S-73 falls within Jun HOB1 and is conserved in Fos HOB1 as a threonine (T-232; Fig. 2). Fos T-232 may also be the target for MAP kinase activity because, as with Jun S-63 and S-73, it is followed by a proline residue and, therefore, conforms to the consensus sequence for MAP kinase (S/TP) (Payne et al. 1991). Preliminary observations suggest that, indeed, T-232 is a substrate for MAP kinase and that the Fos HOB 1/HOB2 domain is responsive to superactivation by $\mathrm{H}$-ras (J.A. Sutherland and A.J. Bannister, unpubl.). It is therefore possible that $\mathrm{H}$-rasinduced phosphorylation of $\mathrm{HOBl}$ regulates the activation functions of both Fos and Jun. This provides an attractive model by which external mitogenic signals, acting through Ras, affect the activation potential of nuclear transcription factors.

The HOB1 and HOB2 motifs may represent binding sites for proteins required to mediate the activation process. Phosphorylation by MAP kinase may potentiate the binding of such proteins to HOB1 (but not HOB2). Regulating one of the activation modules ( $\mathrm{HOBl}$ ) would then leave the second module (HOB2) free to cooperate with a distinct module within the same protein such as that present at the carboxyl terminus. Interestingly, the carboxy-terminal domain of Fos that cooperates with HOB2 is phosphorylated by protein kinase A (Tratner et al. 1992). Regulation by phosphorylation may turn out to be a common feature of activation modules that cooperate with HOB2. The use of such regulation may explain why HOB2 is found cooperating with distinct, rather than identical, modules within a given protein.

Pertinent to this discussion is the conservation of the $\mathrm{HOB} 1$ and HOB2 motifs within other members of the Jun, Fos, and C/EBP families. Both motifs are conserved in the two Jun family members Jun-B and Jun-D. In Jun$B$, however, the proline residue that follows the MAP kinase-phosphorylated residues S-63 and S-73 is not conserved. This may reflect the fact that a kinase, other than the "proline-specific" MAP kinase is responsible for phosphorylating these serines in Jun-B. This would then allow Jun-B to be regulated independently of Jun and Jun-D.

In contrast to Jun family members, the Fos-related proteins, Fra- 1 and Fos-B, do not have a conserved $\mathrm{HOB} 1$ and HOB2 motif. The HOB1/HOB2 region is unique to the Fos protein, suggesting that the activity residing within this domain may distinguish Fos function from that of other Fos-related proteins. The HOB2 motif is highly conserved in all members of the C/EBP family (Williams et al. 1991). This is particularly striking as this family does not show extensive conservation outside its DNAbinding domain. It is interesting to note that the three proteins shown here to possess a HOB2 activation domain, Fos, Jun, and C/EBP, are all members of the bZIP family of DNA-binding proteins. Although this sample is too small to be significant, the possibility that this domain is specific to members of the bZIP family cannot be excluded.

The HOB2-containing activation domains of Fos, Jun, and $\mathrm{C} / \mathrm{EBP}$ show some interesting similarities that may reflect common function. Deletion analysis of these proteins indicates that the HOB2-containing activation domain is less active in the context of the full-length proteins (Friedman and McKnight 1990; Baichwal et al. 1991; Fig. 31. This suggests that a repressive activity may regulate the function of these domains. In the case of Jun, a cell-specific repressor has been implicated in the regulation of the Jun $\mathrm{HOB} 1 / \mathrm{HOB} 2$ domain. It has been proposed that H-ras-induced superactivation of this domain is a result of relief from repressor activity (Baichwal et al. 1991). The results of the Fos deletion analysis imply that the cooperative activity between the HOB2 and the Fos carboxyl terminus is masked completely in the presence of amino-terminal sequences (Fig. 3, lanes $2,4)$. This may be an indication that in $1 \mathrm{BR}$ cells, Fos HOB2 cooperates with HOB1 but not with the carboxyterminal domain, owing to the presence of an inhibitor that specifically prevents cooperation with the carboxyterminal domain.

Analysis of the secondary structure predictions for Fos and Jun reveal that the $\mathrm{HOBl}$ motif is present partly within an $\alpha$-helical region. An $\alpha$-helix is predicted for residues 75-82 of Jun and 234-241 in Fos (Fig. 6). This helix falls just after the S/TP sequence that forms part of the recognition site for MAP kinase. Within this potential helix lie the two conserved glutamic acid residues (E-234 and E-236 in Fos, E-75 and E-77 in Jun) that are required for activation. In addition, the general character of the residues within this helix is conserved between Fos and Jun.

The HOB2 motif is also predicted to lie within an $\alpha$-helix (Fig. 6). In the case of Jun and C/EBP, the entire region is $\alpha$-helical, whereas in Fos, a central core (bounded by two prolines) is predicted to be within an $\alpha$-helical structure. This central core contains the most conserved residues in this motif and has the consensus $F$, $\mathrm{X}, \mathrm{D} / \mathrm{E}, \mathrm{X}, \mathrm{F} / \mathrm{L}, \mathrm{F} / \mathrm{L}$. The first and fifth positions of the core motif are hydrophobic and are predicted to fall on the same face of the potential $\alpha$-helix.

Activation domains characterized so far appear to be related merely by the prevalence of certain residues, and they are categorized as such. Families of activators are exemplified by the glutamine-rich domain of SP1 (Courey and Tjian 1988), the acidic domain of VP16 (Triezenberg et al. 1988), the proline-rich domain of CTF (Mermod et al. 1989), and the serine-threonine-rich domain of Oct-1 (Tanaka and Herr 1990). Transcription factors that fall into each one of these categories do not show absolute sequence identity. Furthermore, there is no evidence that any of these loosely related activation domains are related in function. In contrast, the HOB2 domain of Fos, Jun, and C/EBP shows precise sequence conservation, is similar in predicted secondary structure, and functions in a cooperative manner. Using the HOB2 
homology as a basis, it has been possible to identify successfully a transcription factor (C/EBP) with a cooperating HOB2 domain. The HOB2 motif therefore defines a new class of modular activation domain that can be identified through sequence homology. This motif can now be used to identify other transcription factors with modular activation domains. Cooperation assays with the prototype Fos or Jun HOB motifs could then be used to establish the validity of the observed homologies.

\section{Materials and methods}

\section{Recombinant DNA}

The Fos protein and the various deletions were cloned in-frame with the carboxyl terminus of the GAL4 DNA-binding domain present within the plasmid pHKG, which has the SV40 promoter and polyadenylation site, a polylinker $3^{\prime}$ to the carboxyl terminus of the GAL4 DBD and carries on Fl origin of replication (C. Hagemeier and T. Kouzarides, in prep.). The rat c-fos sequence was used (Curran et al. 1987). The deleted versions 53-380 and 103-380 were generated using the naturally occurring BglII and HincII sites, respectively. To generate the remaining deletions, restriction sites were introduced into the c-fos gene. Three mutagenic oligonucleotides (TK67, TK68, and TK69| were used to introduce the following restriction sites: a BamHI-EcoRI site at positions 749-754 and 756-761, respectively (TK67), a BamHI-SacI site at positions 1061-1066 and 1068-1073, respectively (TK68), and a BamHI-EcoRI site at positions 869-874 and 876-881, respectively (TK69). Mutagenesis was carried out as described previously (Kouzarides et al. 1991). The deletions were generated in the following way: GF 53-308 with SacI of TK68, GF 53-205 with EcoRI of TK67, GF 210-380 with EcoRI of TK67, GF $314-380$ with BamHI of TK68. GF 210-308 contains sequences from EcoR-SacI of TK67 and TK68, GF 210-244 contains sequences from EcoRI-EcoRI of TK67 and TK69, GF 250-308 contains sequences from EcoRI-Sacl of TK69 and TK68. To separate the HOB1 and HOB2 motifs of Jun, two adjacent restriction sites were introduced (a BamHI site at 669674 and an EcoRI site at 676-681) using oligonucleotide TK90 (Angel et al. 1988). These two sites are at precisely the same relative position as the sites described by oligonucleotide TK69 (Fig. 1), which were used to separate the HOBl and HOB2 motifs of Fos. Consequently, the distance between the two motifs in GFHI/JH2 and GJHI/FH2 is identical to that found in Fos and Jun. The GFH2/JH2 construct has Fos sequences from the EcoRI site of TK67 to the BamHI site of TK69 and Jun sequences from the BamHI site of TK90 to a Nael site after residue 193. The GJH1/FH2 clone has Jun sequences from residue 1 of Jun to the EcoRI site of TK90 and Fos sequences from the EcoRI site of TK69 to the EcoRI sites of TK68.

To generate the carboxy-terminal truncations of the Fos protein, a mutagenic oligonucleotide, TK104, was used to generate an EcoRI site and an XbaI site at positions 1130-1135 and 11381143 , respectively. Constructs containing sequences from residue 267-380 were generated using PCR, and deletion at residue 332 was achieved using the $X b a I$ site of TK104.

Domain analysis of C/EBP was accomplished using specific PCR primers to amplify the appropriate domains. To generate the $\mathrm{G}$ )H1/CH2 chimera, a mutagenic oligonucleotide (TK100) was used to introduce a $B a m H I$ and an EcoRI site at positions $712-719$ and $720-726$ in c-jun, respectively. The 29 -residue $\mathrm{HOB} 2$ domain of C/EBP (amplified by PCR/ was fused to Jun at the BamHI site of Jun/TK100. Thus, the distance between Jun $\mathrm{HOB} 1$ and $\mathrm{C} / \mathrm{EBP} \mathrm{HOB} 2$ is the same as the distance between Jun HOB1 and HOB2.
Dimerization of $\mathrm{HOBl}$ was accomplished by introducing the BamHI fragment described by TK67-TK69 into pHKG 210-244. Dimerization of HOB2 was accomplished by introducing the BamHI fragment described by TK 69 and TK68 into PHKG 250 308.

\section{Transfections}

Approximately $1 \times 10^{6} \mathrm{l}$ BR cells [SV40 T antigen-transformed human skin fibroblasts (Mayne et al. 1986)] grown at $37^{\circ} \mathrm{C}(5 \%$ $\mathrm{CO}_{2}$ ) in Dulbecco's Eagle medium supplemented with $10 \%$ fetal calf serum were transfected with a total of $7.5 \mu \mathrm{g}$ of DNA using the calcium-phosphate coprecipitation technique. The precipitate was washed $6 \mathrm{hr}$ after transfection and the cells were harvested $24 \mathrm{hr}$ after transfection. Extracts from these cells were then used for CAT assays and for Western blots. The CAT assays were quantitated by liquid scintillation counting. The Western blots were probed with an antibody against the GAL4 DNA-binding domain (gift of M. Ptashne) to check the level of protein expressed by each vector. All plasmids expressed proteins at relatively equivalent levels. Each transfection was repeated a minimum of two times.

\section{Acknowledgments}

We thank Mark Ptashne for the GAL4 DBD-specific antibody and David Cryer for help with photography. We are grateful to Christian Hagemeier and Steve Jackson for helpful discussion and critical evaluation of the manuscript. This work was funded by the Cancer Research Campaign.

The publication costs of this article were defrayed in part by payment of page charges. This article must therefore be hereby marked "advertisement" in accordance with 18 USC section 1734 solely to indicate this fact.

\section{References}

Abate, C., D. Luk, E. Gagne, R.G. Roeder, and T. Curran. 1990. Fos and Jun cooperate in transcriptional regulation via heterologous activation domains. Mol. Cell. Biol. 10: 55325535.

Abate, C., D. Luk, and T. Curran. 1991. Transcriptional regulation by Fos and Jun in vitro: Interaction among multiple activator and regulatory domains. Mol. Cell. Biol. 11: 3624 3632.

Angel, P., E.A. Allegretto, S.T. Okino, K. Hattori, W.J. Boyle, T. Hunter, and M. Karin. 1988. Oncogene Jun encodes a sequence-specific trans-activator similar to APl. Nature 332: 166-171.

Angel, P., T. Smeal, J. Neek, and M. Karin. 1989. Jun and v-Jun contain multiple regions that participate in transcriptional activation in an independent manner. New Biol. 1: 35-43.

Baichwal, V.R. and R. Tjian. 1990. Control of c-Jun activity by interaction of cell-specific inhibitor with regulatory domain $\delta$ : Differences between v- and c-Jun. Cell 63: 815-825.

Baichwal, V.R., A. Park, and R. Tiian. 1991. v-Src and E J Ras alleviate repression of c-Jun by a cell-specific inhibitor. $\mathrm{Na}$ ture 352: 165-168.

Binétruy, B., T. Smeal, and M. Karin. 1991. Ha-ras augments c-Jun activity and stimulates phosphorylation of its activation domain. Nature 351: 122-127.

Bohmann, D. and R. Tjian. 1989. Biochemical analysis of transcriptional activation by /un: Differential activity of $\mathrm{c}$ - and v-lun. Cell 59: 709-717.

Chiu, R., P. Angel, and M. Karin. 1989. Jun-B differs in its bio- 
logical properties from and is a negative regulator of c-Jun. Cell 59: 979-986.

Courey, A.J. and R. Tjian. 1988. Analysis of SPl in vivo reveals multiple transcriptional domains, including a novel glutamine-rich activation motif. Cell 55: 887-898.

Cousens, D.J., R. Greaves, C.R. Goding, and P. O'Hare. 1989. The C-terminal 79 amino acids of the herpes simplex virus regulatory protein, $\mathrm{Vmw} 65$, efficiently activate transcription in yeast and mammalian cells in chimaeric DNA-binding proteins. EMBO J. 8: 2337-2342.

Curran, T., M.B. Gordon, K.L. Rubino, and L.C. Sambucetti. 1987. Isolation and characterisation of the $c$-fos (rat) cDNA and analysis of post-translational modification in vitro. Oncogene 2: 80-84.

Friedman, A.D. and S.L. McKnight. 1990. Identification of two polypeptide segments of CCAAT/enhancer-binding protein required for transcriptional activation of the serum albumin gene. Genes \& Dev. 4: 1416-1426.

Gentz, R., F.J. Rauscher III, C. Abate, and T. Curran. 1989. Parallel association of Fos and Jun leucine zippers juxtaposes DNA binding domains. Science 243: 1695-1699.

Halazonetis, T.D., K. Georgopoulos, M.E. Greenberg, and P. Leder. 1988. c-Jun dimerizes with itself and with c-Fos, forming complexes of different DNA binding affinities. Cell 55: $917-$ 924.

Kouzarides, T. and E. Ziff. 1988. The role of the leucine zipper in the fos-jun interaction. Nature 336: 646-651.

. 1989a. Leucine zippers of fos, iun and GCN4 dictate specificity of dimerisation and thereby control DNA binding. Nature 340: $568-571$.

. 1989b. Behind the fos and jun leucine zipper. Cancer Cells 1: 71-76.

Kouzarides, T., G. Packham, A. Cook, and P.J. Farrell. 1991. The BZLFl protein of EBV has a coiled coil dimerisation domain without a heptad leucine repeat but with homology to the C/EBP leucine zipper. Oncogene 6: 195-204.

Landschulz, W.H., P.F. Johnson, and S.L. McKnight. 1988a. The leucine zipper: A hypothetical structure common to a new class of DNA binding proteins. Science 240: 1759-1764.

Landschulz, W.H., P.F. Johnson, E.Y. Adashi, B.G. Graves, and S.L. McKnight. 1988b. Isolation of a recombinant copy of the gene encoding C/EBP. Genes \& Dev. 2: 786-800.

Mayne, L.V., A. Priestley, M.R. James, and J.F. Burke. 1986. Efficient immortalisation and morphological transformation of human fibroblasts by transfection with SV40 DNA linked to a dominant marker. Exp. Cell Res. 162: 530-538.

Mermod, N., E.A. O'Neil, T. Kelly, and R. Tiian. 1989. The proline-rich transcriptional activator of CTF/NF-1 is distinct from the replication and DNA binding domain. Cell 58: 741-753.

Nakabeppu, Y., K. Ryder, and D. Nathans. 1988. DNA binding activities of three murine Jun proteins: Stimulation by Fos. Cell 55: 907-915.

Payne, D.M., A.J. Rossomando, P. Martino, A.K. Erickson, J.-H. Her, J. Shabanowitz, D.F. Hunt, M.J. Weber, and T.W. Sturgill. 1991. Identification of the regulatory phosphorylation sites in pp42/mitogen-activated protein kinase (MAP kinase). EMBO J. 10: 885-892.

Pulverer, B.J., J.M. Kyriakis, J. Arruch, E. Nikolakaki, and J.R. Woodgett. 1991. Phosphorylation of c-Jun mediated by MAP kinases. Nature 353: 670-674.

Sassone-Corsi, P., L. Ransone, W.W. Lamph, and I.M. Verma. 1988. Direct interaction between Fos and Jun nuclear oncoproteins: Role of the "leucine zipper" domain. Nature 336: 692-695.

Schuermann, M., M. Neuberg, J.B. Hunter, T. Jenuwein, R.-P.
Ryseck, R. Bravo, and R. Müller. 1989. The leucine repeat motif in Fos protein mediates complex formation with Jun$\mathrm{APl}$ and is required for transformation. Cell 56: 507-516.

Schütte, J., J. Viallet, M. Nau, S. Segal, J. Fadorko, and J. Minna. 1989. Jun-B inhibits and c-Fos stimulates the transforming and transactivating activities of c-Jun. Cell 59: 987-997.

Sellers, I.W. and K. Struhl. 1989. Changing Fos oncoprotein to a Jun-dependent DNA-binding protein with GCN4 dimerisation specificity by swapping "leucine zippers." Nature 341: 74-76.

Smeal, T., B. Binétruy, D.A. Mercola, M. Birrer, and M. Karin. 1991. Oncogenic and transcriptional cooperation with $\mathrm{Ha}$ ras requires phosphorylation of C-Jun on Serines 63 and 73. Nature 354: 494-496.

Tanaka, M. and W. Herr. 1990. Differential transcriptional activation by Oct-1 and Oct-2: Independent activation domains induce Oct-2 phosphorylation. Cell 60: 375-386.

Tratner, I., R. Ofir, and I.M. Verma. 1992. Alteration of a cyclic AMP-dependent protein kinase phosphorylation site in the c-Fos protein augments its transforming potential. Mol. Cell. Biol. 12: 998-1006.

Triezenberg, S.J., R.C. Kingsbury, and S.L. McKnight. 1988. Functional dissection of VP16, the trans-activator of herpes simplex virus immediate early gene expression. Genes \& Dev. 2: 718-729.

Turner, R. and R. Tjian. 1989. Leucine repeats and an adjacent DNA binding domain mediate the formation of functional c-Fos-c-Jun heterodimers. Science 243: 1689-1694.

Williams, S.C., C.A. Cantwell, and P.F. Johnson. 1991. A family of C/EBP-related proteins capable of forming covalently linked leucine zipper dimers in vitro. Genes \& Dev. 5: 15531567. 


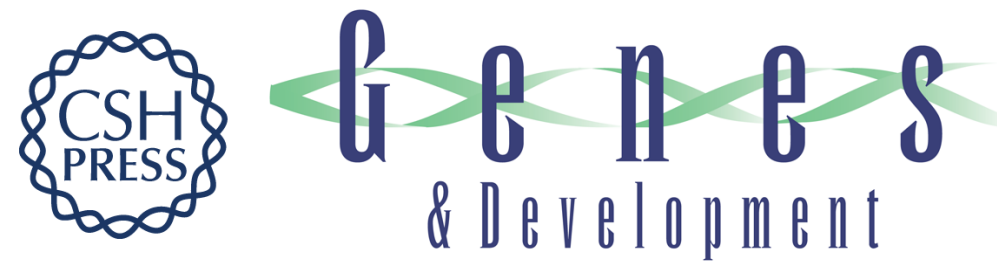

\section{Conserved motifs in Fos and Jun define a new class of activation domain.}

J A Sutherland, A Cook, A J Bannister, et al.

Genes Dev. 1992, 6:

Access the most recent version at doi:10.1101/gad.6.9.1810

References This article cites 36 articles, 9 of which can be accessed free at:

http://genesdev.cshlp.org/content/6/9/1810.full.html\#ref-list-1

License

Email Alerting

Service

Receive free email alerts when new articles cite this article - sign up in the box at the top right corner of the article or click here.

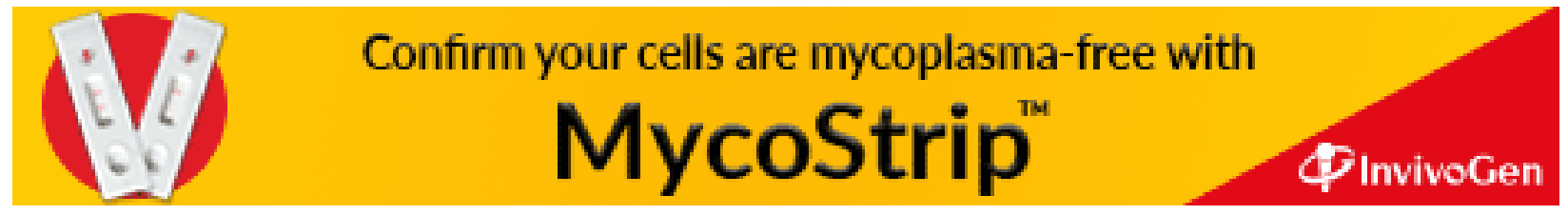

